Rudolf Lüscher

\title{
Die linke Ecke des Wiener Kreises
}

Bemerkungen zu A. Beckermanns „Logischer Positivismus und radikale Gesellschaftsreform", (Analyse \& Kritik 1/79)*

\begin{abstract}
Beckermann's belief in a "direct connection" between Logical Empiricism and socialist politics is unjustified: (I) Logical Empiricism supports - if anything - 'rational', i.e. non-metaphysically grounded political positions, including non-socialist and authoritarian ones. (II) Logical Empiricism offers instruments to anybody willing to talk rationally about politics, but it cannot urge anybody to become politically active. (III) The only systematic attempt to develop a politically relevant sociology within the Vienna Circle, i.e. Neurath's, is at best methodologically inconsistent and at worst pro-authoritarian.
\end{abstract}

Beckermann will das Vorurteil „kritisch engagierter Sozialwissenschaftler“ korrigieren, „Positivisten (seien) mit ihrer Tatsachengläubigkeit allemal Apologeten der bestehenden Verhältnisse“ $(1979,30)$. Man könnte also eine Nachprüfung der „kritischen" Einwände gegen den Logischen Empirismus erwarten (z.B. Horkheimer 1968; Marcuse 1939), denen zufolge die Verbindung von Empirismus, Sinnkritik (und nonkognitivistischer Ethik) schon im Ansatz radikale Gesellschaftskritik verunmögliche. Was allerdings folgt, ist der Nachweis, daß einige Logische Empiristen sich zum Sozialismus bekannten - was Horkheimer schon vor zweiundvierzig Jahren einräumte $(1968,134)$. Beckermann verstärkt diesen Nachweis um den zusätzlichen, die linken Logischen Empiristen hätten eine ,innere Verwandtschaft" (Carnap 1961, XX) zwischen ihrem logisch-empiristischen und ihrem sozialistischen Engagement gespürt. Das ist schon mehr, als Horkheimer zugeben mochte (1968, 134), aber noch recht wenig, weil verwandtschaftliche Beziehungen, die nur gespürt, aber nicht im Familienrodel ausgewiesen werden, nicht nur in Erbschaftsangelegenheiten wenig hergeben. Hegselmann (1979a, 32 ff.; 1979b, 3.1.-3.3.; 1979c) reduziert denn auch die Verwandtschafts- auf eine Nachbarschaftsbeziehung: die linken Logischen Empiristen seien Sozialisten geworden, weil ihr Hauptfeind der österreichische Klerikofaschismus war, dem sich nur die Sozialdemokratie entgegenstellte. Hegselmann läßt auch einiges offen; immerhin gab es in Österreich der Zwischenkriegszeit nicht nur Klerikofaschisten und Sozialdemokraten, sondern auch Liberale und Kommunisten. Was machte denn die österreichische Sozialdemokratie so besonders anziehend, deren Theoretiker sich z.T. erst noch ausdrücklich gegen den Logischen Empirismus wandten? (Vgl. etwa Adler 1936, 197f.) In einem hat Hegselmann aber gewiß recht: wenn die Logischen Empiristen auch von der guten Absicht erfüllt waren, Logischen Empirismus und Sozialismus zusammenzubinden,

* Für Einwände danke ich H. Grünberger und J. B. Neidhart herzlich. 
dann genügt die gute Absicht nicht, das Forschungsprogramm des Logischen Empirismus zu einem pro-sozialistischen zu machen. Beckermanns Bemerkung (1979, 45 Fn. 8) zu Hegselmann (1979a, 32 ff.) geht darauf nicht ein, sondern verbeißt sich in das ebenso unbezweifelbare wie hier unzulängliche Argument, die Logischen Empiristen hätten aber eben die gute Absicht gehabt, und darum seien sie Logische Empiristen geworden. Man kann da nur etwas hilflos fragen, warum sie nicht gleich Marxisten geworden seien.

Beckermann liebäugelt mit einer Vermutung, die auszusprechen ihn seine Gewissenhaftigkeit dann doch hindert:

(1) Das Forschungsprogramm des Logischen Empirismus ist geeignet, eine sozialistische politische Option zu begründen.

Er behilft sich mit der spiegelverkehrten Vermutung, eine sozialistische politische Option sei geeignet, zum Logischen Empirismus zu motivieren, läßt aber auch diese Vermutung in der Schwebe, weil er nicht recht damit heraus will, ob die linken Logischen Empiristen als Sozialisten motiviert waren, ein Sinnkriterium auszubilden, oder als Logische Empiristen motiviert waren, die Ausbeutung der Arbeiterklasse zu bekämpfen.

Beckermann läßt reichlich offen, ob die linken Logischen Empiristen auf eine, sozialistische' oder auf eine, rationale' Gesellschaftsordnung zielten. In seiner Verwendung von ,rationale Gesellschaftsordnung' ist von der Neuen Sachlichkeit über den Eudämonismus über die Vollsozialisierung über die weltzugewandte Philosophie bis zum Sozialismus alles irgendwie da. Ich schlage vor, (1) etwas aufzusplittern. Wenn wir davon ausgehen, daß eine politische Bewegung sich (a) entweder rational oder metaphysisch legitimiert, (b) entweder eine demokratische oder eine autoritäre Staatsorganisation anstrebt und endlich (c) entweder eine formaldemokratische (ausgegrenzter politischer Sektor mit gleichem Stimmrecht, keine demokratischegalitären Forderungen für Produktionsbereich und Ressourcenverteilung) oder eine materialdemokratische (gleiches Stimmrecht in allen gesellschaftsrelevanten Entscheidungsbereichen, egalitäre Produktions- und Verteilungsstruktur) Staats- bzw. Gesellschaftsorganisation fordert, dann wird (1) zu:

(1*) Das Forschungsprogramm des Logischen Empirismus ist geeignet, folgende politische Optionen zu begründen:

rational-formaldemokratisch

rational-materialdemokratisch

rational-autoritär.

Diese Abschwächung von (1) läßt den Logischen Empirismus noch weniger sozialistisch-demokratisch ausgerichtet erscheinen, als selbst Hegselmann (1979c) annimmt. Man kann sie so begründen: Die erste Hürde, die eine Theorie nehmen muß, welche sich einer politischen Option zuordnen läßt, ist im logisch-empiristischen Prüfungsgang die der Metaphysikfreiheit ihrer Rechtfertigungsbasis. Ganz unabhängig davon, was für Organisations-, Verwaltungs-, Entscheidungsmuster eine politische Theorie vorschlägt, kann sie vor dem Logischen Empirismus nur dann bestehen, wenn sie ihre Vorschläge nicht mit theologischen, geschichtsmetaphysischen usw. Scheinargumenten absolutistisch absichert. Eine materialdemokratische 
Option, die sich auf die Bibel berufen wollte, hätte keine Chancen. Auch jede Variante des Marxismus, die sich nicht in die deterministische Prognose zusammenzieht, der nächste Zusammenbruch komme bestimmt, hätte keine Chance (Da setzt dann Neuraths rationale Rekonstruktion der Marx'schen Theorie an). Umgekehrt hat eine metaphysikfreie politische Option, die antidemokratische Strukturen anstrebt, vor dem Logischen Empirismus Chancen. Das Argument, auf das Beckermann sich beruft $(1979,37)$ : die Erkenntnistheorie schließe alle privilegierten $\mathrm{Zu}$ gänge zur Erkenntnis aus und anerkenne nur diskursive Begründungen, also habe der Logische Empirismus eine Affinität zur Demokratie, ist zu schwach: (a) Auch wenn man dieses Argument für politisch legitimationsrelevant ansieht, können aus ihm keine Gründe gegen eine politische Option gemünzt werden, die z. B. eine autoritäre Organisation der Produktion diskursiv begründet (Man braucht sich nur an Taylors Feststellung zu erinnern: natürlich könnten die Arbeiter, wenn sie Zeit hätten, ihre Arbeit selbst organisieren und den Rationalisierungsfachmann einsparen. Aber sie hätten eben keine Zeit, und wenn sie sich welche nähmen, bräche die Produktion zu ihrem eigenen Schaden zusammen). (b) Es fällt schwer zu sehen, wie aus der These, es gebe keine privilegierten Erkenntnissubjekte, die - Forderungen begründende - These herausgepreßt werden sollte, alle Arten von Privilegierungen seien irrational. Selbst wenn man die methodologische These empirisch nehmen wollte - wir haben alle die gleichen Erkenntnischancen -, fehlt die Brücke zur - Forderungen begründenden - These, nur Arbeits- und Rollenteilungen, in denen alle Rollen gleiche z. B. Entscheidungsprivilegien tragen, wären rational.

Ich sehe nicht, wie man ohne empirische Zusatzannahmen, allein auf der Basis der methodologischen Regelungen des Forschungsprogramms, dem Logischen Empirismus zutrauen könnte, er sei besser geeignet, demokratische als autoritäre Optionen auszuzeichnen. $\mathrm{Daß}$ er für die Auszeichnung materialdemokratischer Optionen nicht besonders geeignet ist, halten Hegselmann (1979b, 3.1.-3.3.; 1979c) und Köhler $(1979,58)$ fest; daß er eine starke Affinität zu formaldemokratischen Optionen hätte, scheint mir zweifelhaft. Aus (1) würde dann

$\left(1^{*}\right)$ Das Forschungsprogramm des Logischen Empirismus ist geeignet, rationale politische Optionen zu begründen.

Das führt zu einer zweiten Frage: wie soll denn die Verwandtschaft zwischen Forschungsprogramm und politischem Engagement aufgefaßt werden? Ich sehe drei Möglichkeiten:

(2) Was ein Logischer Empirist neben seiner Forschungsarbeit tut, ist seine Sache (solang er nicht Spiritualist, Faschist oder Katholik wird).

$\left(2^{*}\right)$ Vertreter des Logischen Empirismus, die sich politisch engagieren, werden ihr Engagement in Formen begründen und verteidigen, die den diskurssteuernden Regeln des Logischen Empirismus gerecht werden.

(2**) Logische Empiristen werden durch das Forschungsprogramm des Logischen Empirismus zu einem sozialistischen politischen Engagement angehalten, und sie werden ihr Engagement in Formen begründen und verteidigen, die den diskurssteuernden Regeln des Logischen Empirismus gerecht werden. 
(2) - oder eine noch schwächere Version von (2), ohne den eingeklammerten Halbsatz - dürfte dem entsprechen, was der konservative Flügel des Wiener Kreises akzeptiert hätte. Wenn jemand Logischer Empirist wird, geht er nicht eine Verpflichtung ein, sein (Alltags)leben so einzurichten, daß er allem metaphysisch Angehauchten aus dem Wege geht oder es gar bekämpft. Jedenfalls könnte eine solche ,Verpflichtung' nur die Form einer de facto von allen Logischen Empiristen anerkannten Lebensregel annehmen, für die sich zwar Gründe angeben lassen, die aber keinen absolutistischen Zwangscharakter vom Forschungsprogramm verliehen erhält.

$\left(2^{* *}\right)$ dürfte etwa dem entsprechen, was Beckermann sich wünscht. $\left(2^{* *}\right)$ ist aber, selbst wenn man ,sozialistisch' - wegen $\left(1^{* *}\right)$ - in ,rational' mildert, zu stark. Was sich etwa anbietet, wäre folgende Abschwächung: Wenn jemand Logischer Empirist wird, dann weil er in der Wissenschaft und im Alltag rational handeln will, und (a) wer rational handeln will, wird immer versuchen, das zu tun, was sich am besten begründen läßt, und (b) die Entscheidung, sich für rationale politische Bewegungen zu engagieren, läßt sich besser begründen als die Entscheidung, sich für irrationale politische Bewegungen zu engagieren oder sich politisch gar nicht $\mathrm{zu}$ engagieren. Eine solche Verknüpfung von Feststellungen über die Motivation, mit der jemand Logischer Empirist wird, und begründungsfähigen Konsequenzen, die diese Motivation für einen Logischen Empiristen hätte, wäre m. E. das Stärkste, was linke Logische Empiristen anbieten könnten. Auch dafür wären freilich empirische Zusätze erforderlich, die die Rationalität des Engagements belegen. Aber, so scheint es, diese stärkste ideologiefreie Konstruktion genügt den linken Logischen Empiristen nicht ganz. Im Grunde sollte der Logische Empirist dort, wo er engagiert auftritt, sich bemühen, Begründungen als Entscheidungshilfen und -vorbereitungen anzubieten: einen nicht überredungshungrigen Pluralismus zu vertreten: ,Wenn du das tust, werden die Folgen eintreten, wenn du jenes tust, jene - was du tun willst, mußt du selbst entscheiden.' Damit haben die linken Logischen Empiristen nichts im Sinn. Metaphysiker, so hören wir, leiden an einer ,Krankheit" (Hahn 1930, 24) und fixen „Narkotika“ (Carnap 1934, 260). Wo der linke Wiener Kreis militant wird, kommt er den Gegnern psychiatrisch. Guten Gewissens, versteht sich: die Gegner reden Sinnlosigkeiten - sie reden eigentlich gar nicht, sie delirieren und gehören behandelt. Die polemische Rhetorik arbeitet hinterhältig: sie nutzt deskriptiv klingende Begriffe, wo sie nach eigener Sprachregelung die Deskription längst zugunsten nichtkognitiver Sprechweisen verlassen müßte; sie gibt sich diagnostisch, wo sie Vorschriften im Sinne hat. Natürlich, sagt der linke Wiener Kreis, dürft ihr Narkotika konsumieren wollen. Das ist eure Entscheidung. Wir konsumieren keine, weil wir die Gründe dagegen kennen. Das ist unsere Entscheidung. Aber daß ihr, auch wenn ihr unsere Gründe gehört habt, weiter fixen wollt, das ist keine Entscheidung mehr, das ist schon ein Symptom (vgl. Carnap 1934, 259; Carnap/Hahn/Neurath 1929, 17). In Neuraths Sozialbehaviorismus kommt derlei Polemik dann auf ihren wissenschaftlichen Ausdruck: Metaphysik ist ein konditionierter Reflex (Freilich mit dem paradoxen Komplement: der Logische Empirismus auch. s. u. (4)). Soìche Psychiatrisierung des Gegners ist, denke ich, kein bloßes Stilproblem. In ihr tritt ein 
Defizit zutage, das der Logische Empirismus nicht decken kann: als Kontrollinstrument vernünftiger Rede ist er nicht in der Lage, zu vernünftigem Reden zu motivieren. Er hilft nur dem, der sich schon helfen lassen will. Diesen Willen zu wecken, sind „Beeinflussung, Erziehung“ (Carnap 1934, 258), „Aufruf, Erziehung, Vorbild“ (Carnap 1934, 260) bestimmt. Sie können ihrerseits nicht wieder in Begründungszusammenhänge eingezogen werden; und wo sie nicht greifen, bleibt nur die Zuspitzung der Erziehung zur abrichtenden Beeinflussung. Wenn man diese Konstruktion mit Neuraths Sozialbehaviorismus zusammennimmt - und warum sollte man das nicht? - wird das politische Engagement des Logischen Empirismus zu einer reichlich finsteren Angelegenheit. Die eigentümliche Ohnmacht der diskursiven Orientierung: für die Ohren der Bekehrten zu predigen, wird dort bedrohlich, wo sie auf den Gegner einwirken will, und keine rechtfertigungsfähige Rhetorik zwischen der Beeinflussung und dem Begründungsverfahren finden kann; da gerinnt ihr die Rhetorik leicht und unkontrollierbar zum Abrichtungsversuch. Vermutlich kommt jene Bedrohlichkeit davon, daß der linke Logische Empirismus Ideologiekritik nach dem Pfaffentrug-Muster strickt, das er mit mechanistischen Annahmen über die Wirkung von Ideologien verknüpft (vgl. Carnap/Hahn/Neurath 1929, 17, 29). Für ihn besteht die ideologiekritische Aufgabe darin, den ,Schutt der Jahrtausende“ (29) wegzuräumen. Wo Ideologiekritik zur Müllabfuhr wird, gibt sie die Chance preis, an den Ideologien, die sie sinnkritisch wegputzt, das freizulegen und neu zu machen, was auf - etwa - eine ,rationale Gesellschaftsordnung' zielt; stattdessen macht sie tabula rasa und wundert sich, wenn sich nichts mehr rührt. An diesem Punkt setzte die ,,kritische“ Attacke auf den Logischen Empirismus ein - und an diesem Punkt hakt der ,kritische“ Verdacht ein, Tatsachengläubigkeit und Apologetik hätten miteinander etwas zu schaffen: Wenn, so könnte man verkürzend an die ,,kritische“ Position erinnern, der Logische Empirismus die Widersprüche in den gesellschaftlichen Verhältnissen zugunsten tatsachenfixierender Momentaufnahmen objektivierend stillstellt, dann wird er in der Tat die offizielle Version der Wirklichkeit auf die Platte bannen, die auch auf sachliche - im doppelten Sinne - Stillstellung aus ist. Und er wird sich dann jeder Kritikchance entschlagen, die an den gesellschaftlichen Verhältnissen selber einhakt statt an ,sozialen Prognosen“, zu denen man sich entschließen muß (vgl. Neurath 1931, $128 \mathrm{ff}$.):

$\left(2^{*}\right)$ scheint aussichtsreicher. Wenn man das Forschungsprogramm des Logischen Empirismus als Kontrollinstrument begreift, das jemanden, der sein Reden an ihm ausrichtet, davor beschützt, demagogischen, absolutistischen Unsinn zu verbreiten und es ihm ermöglicht - und ihn zwingt - seine Argumentationen so auszulegen, daß sie ohńe Begriffsmogelei und Punkt für Punkt überprüfbar aufgebaut werden: dann kann man $\left(1^{* *}\right)$ und $\left(2^{*}\right)$ zu der Vermutung verbinden, das Forschungsprogramm des Logischen Empirismus sei das geeignete sprachregelnde Instrument für jeden, der sich entschließt, rationale politische Optionen zu vertreten. Man müßte selbst dabei in Kauf nehmen, daß die Bestimmungsrichtung nicht vom Forschungsprogramm zum Engagement läuft, sondern vom Engagement zum Forschungsprogramm. Das würde zwar auf Beckermanns Argument (1979, 45 Fn. 8) gegen Hegselmann passen, wonach man Logischer Empirist wird, wenn man links ist, aber nicht 
auf Beckermanns Annahme, daß man erst recht links bleibt, wenn man Logischer Empirist geworden ist. Es sei denn, die sozialistische Option wäre prinzipiell die am besten begründbare - aber gerade das darf man als Logischer Empirist nicht voraussetzen.

So würde aus Beckermanns Wunschthese (1) die kombinierte Vermutung:

(3) Wenn jemand sich für eine rationale politische Option engagieren und sein Engagement rational begründen will, dann kann er auf das Forschungsprogramm des Logischen Empirismus als Kontrollinstrument seiner Rede zurückgreifen und dieses Forschungsprogramm zugleich zur Begründung seiner und zur Kritik irrationaler politischer Optionen heranziehen.

An (1) gemessen, klingt (3) nicht eben begeisternd. Aber schon wenn man (1) einigermaßen auf logisch-empiristische Regelungen abstimmen wollte, könnte es nicht mehr hergeben als: (a) die sozialistische Option bedarf keiner metaphysischen Absicherung, (b) die besten verfügbaren empirischen Argumente zeichnen die sozialistische Option als diejenige aus, die am besten geeignet ist, diejenigen gesellschaftlichen Ziele zu erreichen, die (c) entweder de facto von all denen geteilt werden, die sich zu rationalen politischen Optionen bekennen oder (d) mit solchen Begründungen abgesichert werden können, die die Zustimmung all jener finden, die sich zu rationalen Optionen bekennen.

Ich möchte nun an Neuraths Rekonstruktion der sozialistischen Option plausibel machen, daß selbst (3) noch zu weit geht, weil der Logische Empirismus offenbar eine Soziologie verkraften kann, die - bzw. deren politische Umsetzung - (a) dem Begriff der rationalen politischen Option den Boden entzieht, (b) die guten Absichten der linken Logischen Empiristen zu konditionierten Reflexen hinabdrückt und (c) logisch-empiristische Annahmen mit der Begründung und Empfehlung einer autoritären Gesellschaftsordnung koppelt:

(4) Das Forschungsprogramm des Logischen Empirismus begünstigt eine reduktionistische Soziologie, die Gefahr läuft, sich in eine Paradoxie zu verstrikken oder dieser Paradoxie um den Preis ihrer Annäherung an die Rechtfertigung einer autoritär-rationalen Gesellschaftsordnung zu entgehen.

Unter den einheitswissenschaftlichen Prämissen des Logischen Empirismus müssen die Daten einer soziologischen Theorie Protokollaussagen über beobachtbare Ereignisse sein. Neuraths ,sozialbehavioristische“ Konstruktion (1931; 1932) macht mit dieser Voraussetzung Ernst. Er unterstellt zusätzlich, daß die Gesetze, mit denen Erklärungen sozialer Ereignisse gegeben werden können, kausale Verknüpfungen zwischen Reizen und Reaktionen angeben. Was traditionellen Theorien ,Subjekt' hieß, scheidet aus $(1931,11)$ : Die empirische Soziologie befaßt sich mit reizgesteuerten Organismen. Sprachliche Äußerungen werden als reizgesteuerte Reaktionen analysiert, die ihrerseits Reaktionen auslösen (1931, VI; 1932, $281 \mathrm{ff}$.). Da eine erkenntnistheoretische Privilegierung des Soziologen ausgeschlossen ist, gerät die Theorie in eine paradoxe Situation: Der konsequent angewendete behavioristische Reduktionismus scheint, wenn er sich auf sich selber anwendet, gar nicht mehr fähig, eine Theorie zu erzeugen, d.i. ein diskursiv kritisierbares Gebilde, das Wahrheitsansprüche anmeldet. Horkheimer hat das zum Anlaß genommen, über 
den „,Gelehrten“ zu spotten, der ,objektiv genug (sei), sich selber als Element vor(zu)stell(en)“ (1968, 103). Der Spott ist ein Zitat aus Neurath $(1931,131)$, den das Reduktionsparadox nicht irritiert hat (Seine seltsame Gleichgültigkeit gegenüber dem Problem, wie man an ein Glied einer Reiz-Reaktions-Verflechtung denn sollte Wahrheitsfragen stellen können, kehrt m.E. in seinen Arbeiten zur visualisierten Statistik wieder, der er zutraut, Erziehung unter Umgehung umgangssprachlicher Verständigung zu leisten (vgl. Neurath 1973, $224 \mathrm{ff}$., $243 \mathrm{ff}$.)).

Neurath räumt ein, die ,soziale Prognostik“" werde sich mit der Prognose singulärer Ereignisse schwer tun $(1931,128-133)$. Aber sein Sozialbehaviorismus sei ja auch kein „Laplaceanismus“ (128-133). Wie in der modernen Physik müsse man in der Soziologie mit mikroindeterministischen Einsprengseln in der makrodeterministischen Theorie auskommen und sich damit abfinden, daß soziologische Prognosen risikobehaftet seien, daß man sich zu ihnen entschließen müsse, und daß die Hypothesen, deren man sich bediene, jeweils nicht die einzig möglichen seien, sondern daß die verfügbaren Daten allemal unter verschiedene vergleichbar solide Erklärungen gebracht werden könnten (vgl. Neurath 1932-33; 1935).

Man könnte versuchen, aus dieser mikroindeterministischen Konzeption eine Lösung des Selbstreduktionsproblems zu ziehen: Was in Situationen prognostischer Unsicherheit geschieht, wird von denen, mit denen es geschieht bzw. die es zustandebringen, nicht in sozialbehavioristische Termini, sondern in traditionelle Äußerungsformen gefaßt - ,ich glaube', ,wir beschließen' usw. Das schließt nicht aus, daß eine (nachträgliche) Erklärung aus den Behauptungen, die da scheinbar gefallen sind, wieder reizsteuernde akustische Reaktionen auf Umweltreize macht, aber diese nachträgliche Erklärung ist für die Situation riskanter Empfehlung und Entscheidung irrelevant. Eine solche Lösung, die darauf hinausliefe, das abgeschaffte Subjekt als Wackelkontakt wieder ins Reiz-Reaktions-System einzubauen, führt gewiß zu Kurzschlüssen.

Aber diese Kurzschlüsse hätten ihren Nutzen, und zwar genau dort, wo die empirische Soziologie in die Nähe des politischen Engagements kommt. Für Neurath ist ausgemacht, daß die besten sozialen Prognosen solchen Organisations- und (Re)produktionsstrukturen die größte Chance geben, die der sozialistischen Programmatik entsprächen. Er zehrt von einem deterministisch-ökonomistischen Marxismus; das verträgt sich gut mit der Anlage der Empirischen Soziologie, in der Marx als jener Soziologe gelobt wird, der noch am wenigsten metaphysische Reste in seiner Theorie belassen habe. Neurath begreift seine Empirische Soziologie als Rekonstruktion des rationalen Kerns der Marx'schen Theorie (1931, VI). Die Ausrichtung an der deterministischen Marx-Interpretation hat den Vorzug, das Reduktionsparadox in den Hintergrund zu schieben: der auch hier lästige ,subjektive Faktor' wiegt umso leichter, je sicherer die deterministische Basis wird. Aber ein voluntaristisches Moment scheint dennoch unverzichtbar - Neurath engagiert sich ja selber aktiv für den Sozialismus, und Neurath räumt ein, soziale Prognosen seien risikobehaftet. Da kommt der Wackelkontakt Subjekt zum Knistern. Wenn zwischen konkurrierenden Hypothesensystemen nicht bloß aufgrund der Datenbasis gewählt werden kann, sondern wenn in ihre Wahl ein Entschlußmoment eingeht, dann kann die (sozialisti- 
sche) Zukunft nicht nur erwartet, dann muß sie geplant werden. Daß Planung möglich sei, ist für Neurath gewiß (vgl. hierzu Hegselmann 1979b, 2.1.-2.2.). Dem "sociologist of happiness" (vgl. M. Neurath in Neurath 1973, 79) ist freilich auch gewiß, daß Planung sich am Glück der verplanten Individuen orientieren müsse (vgl. Neurath 1917; 1925, $100 \mathrm{ff}$.). Die gute Gesellschaft wäre durch ,Sozialepikureismus" charakterisiert $(1925,26$ f.; 1928, 136) - einen Epikureismus aus Solidarität (1928, IX-X;1931, 135). Aber wie sich Neurath gleich befleißigt, ,Solidarität“" zur „Solidarität des Schicksals“ $(1931,135)$ zu naturalisieren, und wie er offen läßt, woran denn unter sozialbehavioristischen Voraussetzungen Glück und Abstumpfung zu unterscheiden wären (vgl. Horkheimer 1968, 110) - so modelt er die Planung der guten Gesellschaftsordnung nach dem Muster der Kriegswirtschaftslehre. Freilich nennt er die „Wirtschaftsordnung des Anarchismus“ $(1925,91)$ als Fernziel. Aber wo geplant werden soll, wären demokratische Entscheidungsprozesse nicht angezeigt (vgl. 1925, passim). Solch straff autoritäre, zentralistische Verwaltung des gesellschaftlichen Glücks wäre beunruhigend genug, aber immer noch mit dem Argument zu dämpfen, Neurath habe eben unter den Bedingungen der verfehlten mitteleuropäischen Revolution noch das beste aus der Lage machen wollen (vgl. 1925, 83). Er baut aber in die Empirische Soziologie selber eine autoritäre Konstruktion ein, die, wenn ich recht sehe, bei seiner Kombination von Sozialbehaviorismus und Mikroindeterminismus unvermeidlich anfällt. Zur Zukunftsplanung sind Neurath zufolge „Gesellschaftstechniker“ (1931) berufen, die möglichst gut begründete Muster guter Vergesellschaftung entwerfen und durchzusetzen suchen. Kollektive, materialdemokratische Ausbildung der guten Gesellschaft faßt Neurath gar nicht ins Auge, und es scheint mir zumindest plausibel, daß die Konstruktion der Empirischen Soziologie gesamtgesellschaftliche Entscheidungsprozesse nur als solche über die Gesamtgesellschaft fassen kann. Die planerischen Kurzschlüsse im Reiz-Reaktions-System muß sie an Individuen zurückbinden, deren Verhalten unter der sozialbehavioristischen Erklärungsschwelle durchläuft - im Gegensatz zu dem der Massen, für deren Verhalten behavioristische Erklärungen zuständig sind. Die Koppelung von Mikroindeterminismus und Makrodeterminismus kann einigermaßen paradoxiefrei vorgenommen werden, wenn man einen Schnitt legt zwischen das Individuum, dessen Entscheidungen - mögen sie noch so sehr Reaktionen sein durch das gezwungenermaßen grobmaschige Prognosenetz einer behavioristischen Soziologie schlüpfen, und die Massen, die gerade die richtige Größenordnung haben, in den Maschen hängen zu bleiben (vgl. 1925, $106 \mathrm{f}$.). Kombiniert man die Figur des Gesellschaftstechnikers mit den Grundannahmen des Sozialbehaviorismus, dann erscheint er als Reizerzeuger, der ohne diskursive Überzeugung auskommt, weil er die Massen lenken kann. Daß Neurath derlei intendiert hätte, kann man ohne Zögern ausschließen. Daß die Empirische Soziologie dem Verdacht, sie wäre geeignet, derlei als machbar vorzuführen und womöglich ein Stück weit zu rechtfertigen, entzogen werden könne - das geht nur über das Argument, sie laufe am Selbstreduktionsproblem auf, sei inkonsistent und insofern harmlos.

Selbst dann wäre die einzige ausgeführte politische Konzeption im Rahmen des Logischen Empirismus eine, die für eine autoritäre Lösung plädiert. Eben daraus 
kann man ein starkes Argument für (1) machen: was wir in der zentralplanwirtschaftlichen Konstruktion vor uns haben, ist eine zur Rechtfertigung des, demokratischen Zentralismus' geeignete Theorie, die die Avantgarde-Modelle der leninistischen Tradition bruchlos dorthin fortschreibt, wo sie zu ihrer Rechtfertigung nichts mehr benötigen als die Zuwachsrate der Elektrifizierung. Weder Neurath noch Beckermann hätten an einer solchen Verteidigung von (1) ihre Freude. Umgekehrt zweifle ich, ob sich angesichts von (4) auch nur (3) noch halten ließe: die sozialbehavioristische Konzeption macht aus dem Begriff der, rationalen politischen Option' Makulatur, und die zentralplanwirtschaftliche staucht, rational' auf ,technisch zweckmäßig، zusammen. Eine linke Kritik am Logischen Empirismus freilich läuft nicht ohne massive Selbstkritik ab; gegen Neurath kann nicht an, wer nicht gegen Avantgarde-Modelle anwill.

Vor einundvierzig Jahren schrieb Karl Korsch (1974, 246 Fn. 138):

Horkheimer will mich natürlich gegen diese Leute (Frank, Neurath usw.) gebrauchen, ich habe aber mehr mit letzteren gemein als mit den Institutsleuten und ihrer importierten Philosophie (...). Andererseits stehe ich als Marxist und als gesellschaftswissenschaftlicher Materialist natürlich auch gegen die beste Form des naturwissenschaftlichen Materialismus. Also wie immer eine auch taktisch schwierige Situation.

Inzwischen geht es nicht mehr um Taktik; schwierig ist die Situation geblieben.

\section{Bibliographie}

Adler, M. (1936), Das Rätsel der Gesellschaft, Wien

Beckermann, A. (1979), Logischer Positivismus und radikale Gesellschaftsreform, in: Analyse \& Kritik 1, 30-46

Carnap, R. (1934), Theoretische Fragen und praktische Entscheidungen, in: Natur und Geist 2, 257-260 (wieder in: Schleichert 1975)

- (1961), Der logische Aufbau der Welt, Hamburg

Carnap, R./Hahn, H./Neurath, O. (1929), Wissenschaftliche Weltauffassung - Der Wiener Kreis, Wien (wieder in: Schleichert 1975)

Hahn, H. (1930), Uberflüssige Wesenbeiten, Wien (wieder in: Schleichert 1975)

Hegselmann, R. (1979a), Normativität und Rationalität, Frankfurt

- (1979b), Otto Neurath, in: O. Neurath, Wissenschaftliche Weltauffassung (R.H. ed.), Frankfurt

- (1979c), Grenzen der wissenschaftlichen Weltauffassung des Wiener Kreises, in: Analyse \& Kritik 1, 47-50

Horkheimer, M. (1968), Der neueste Angriff auf die Metaphysik (1937), in: M. H., Kritische Theorie II, 82-136, Frankfurt

Korsch, K. (1974), Brief an Paul Mattick, 23.12.1938, cit. in: M. Buckmiller/G. Langkau (ed.): K. K., Briefe an P. Partos, P. Mattick und B. Brecht, in: C. Pozzoli (ed.): Jabrbuch Arbeiterbewegung II, Frankfurt

Köhler, W. R. (1979), Zerstört der Logische Empirismus die praktische Rationalität?, in: Analyse \& Kritik 1, 51-59

Marcuse, H. (1939), Rezension der International Encyclopedia of United Science, in: Zeitschrift für Sozialforschung 8, 228-232

Neurath, O. (1919), Das umgekehrte Taylorsystem, in: Durcb die Kriegswirtschaft zur Naturalwirtschaft, 205-208, München

- (1925), Wirtschaftsplan und Naturalrechnung, Berlin

- (1928), Lebensgestaltung und Klassenkampf, Wien 
Neurath, O. (1931), Empirische Soziologie, Wien

Neurath, O. (1932-33), Protokollsätze, in: Erkenntnis 3, 204-214 (wieder in: Schleichert 1975)

Neurath, O. (1932), Sozialbehaviorismus, in: Sociologus 8, 281-288

Neurath, O. (1935), Pseudorationalismus der Falsifikation, in: Erkenntnis 5, 353-365

Neurath, O. (1973), Empiricism and Sociology (M. Neurath/R. S. Cohen ed.), Dordrecht/ Boston

Schleichert, H. (ed.) (1975), Logischer Empirismus - der Wiener Kreis, München 